\title{
e-Migrinter
}

$8 \mid 2012$

Regards sur les migrations sud-asiatiques

\section{Smaïn Laacher, De la violence à la persécution, femmes} sur la route de l'exil

\section{Mickaël Quintard}

\section{(2) OpenEdition}

\section{Journals}

Édition électronique

URL : https://journals.openedition.org/e-migrinter/671

DOI : 10.4000/e-migrinter.671

ISSN : 1961-9685

Éditeur

UMR 7301 - Migrinter

\section{Édition imprimée}

Date de publication : 4 avril 2012

Pagination : 141-143

ISSN : 1961-9685

\section{Référence électronique}

Mickaël Quintard, « Smaïn Laacher, De la violence à la persécution, femmes sur la route de l'exil », eMigrinter [En ligne], 8 | 2012, mis en ligne le, consulté le 20 mai 2021. URL : http://

journals.openedition.org/e-migrinter/671 ; DOI : https://doi.org/10.4000/e-migrinter.671 


\section{Laacher, Smaïn (2010) De la violence à la persécution, femmes sur la route de l'exil, Paris, La Dispute, 142 p.}

\section{Mickaël Quintard}

\section{travers six chapitres structurés autour de récits de femmes migrantes collectés dans quatre} pays ${ }^{1}$, le sociologue Smaïn Laacher interroge l'enjeu des catégorisations du droit d'asile à la lumière d'une thématique encore trop discrète dans la littérature tant institutionnelle que scientifique. Les violences vécues par les femmes - parce qu'elles sont femmes - durant leurs parcours migratoires sont ainsi appréhendées par l'auteur sous le prisme des acteurs, lieux et configurations de ces violences, mais aussi à travers les résistances, recours et perceptions qu'elles génèrent. Le regard est alors particulièrement mobilisé sur les violences sexuelles, instituant une problématique du corps à l'épreuve migratoire. Un problème non reconnu n'ayant que peu de chances d'être résolu, il s'agit pour l'auteur «de rendre publiques ces violences et de contribuer à faire comprendre et reconnaître leur spécificité, qui n'entre pas jusqu'ici dans les catégories institutionnellement reconnues » (p.13).

Dans un contexte de durcissement du droit d'asile européen ${ }^{2}$, d'externalisation des procédures et d'élévation du niveau de la preuve de la persécution, Smaïn Laacher délivre une réflexion critique sur le décalage entre les catégorisations institutionnelles et/ou académiques et les réalités vécues par les migrantes. Il apporte ainsi sa contribution à la mise en lumière d'une utilisation particulière de la rhétorique du relativisme culturel au sein d'une instrumentalisation permanente de l'immigration. Cette critique

\footnotetext{
${ }^{1}$ Algérie, Maroc, Espagne et France.

${ }^{2}$ Symbolisé par la récente condamnation française (02/02/2012) par la Cour européenne des droits de l'homme sanctionnant l'absence de recours suspensif dans la procédure «prioritaire » d'asile.
}

trouve essentiellement son matériel empirique dans les pays maghrébins où le traitement des procédures relatives au droit d'asile prend corps dans des contextes politiques marqués par la persistance des représentations de l'étranger comme « figure trouble » (p.20) et la relative inexistence d'un espace public pouvant permettre un mouvement de réclamation de la société civile au nom de l'étranger.

Au-delà d'avoir affaire à un système de protection historiquement situé, restreint et partisan - traduisant alors une double discrimination $^{3}$-, les migrantes sont en prise avec une institution dont les évaluations s'organisent en fonction de typologies basées sur des considérations nationales et territoriales. Face à des catégorisations institutionnelles qui distinguent, hiérarchisent et désunissent les différentes formes de violences les unes des autres, l'auteur oppose une grille de lecture restituant l'individu dans la totalité de sa condition et de sa vulnérabilité.

L'impossibilité pratique d'isoler une forme de violence particulière est ainsi théoriquement dépassée à travers la notion de "continuum de violence», permettant ainsi de penser les violences indépendamment des frontières traversées et les protections avant, pendant et après le voyage: "Ne pas analyser ensemble les espaces de production taxinomiques et leur traduction dans les histoires singulières et collectives, c'est s'interdire de comprendre la complexité de ces situations, (...) leur spécificité et leurs conséquences sociales » (p.57).

${ }^{3} \mathrm{En}$ tant que demandeuses d'asile et en tant que femmes. 
Du point de vue académique, la dichotomie migration forcée/migration volontaire est supplantée par une traduction des situations de départ en termes de "contrainte rationalisée », déclinée ensuite en « choix volontaires ». L'auteur souligne la difficulté de penser des problématiques plus spécifiques comme la traite des êtres humains et la prostitution à travers la grille de lecture migration forcée/migration volontaire, dont la simplification excessive ne reflète pas la difficulté à distinguer clairement ces deux catégories de migration.

Dans l'analyse de ces odyssées, Smaïn Laacher nous amène sur les itinéraires des migrantes et dans les espaces de la violence. Des espaces de départs aux espaces d'arrivées en passant par ceux du voyage, la violence se donne à lire à travers l'ensemble des lieux de circulation comme un élément à la fois constant et diffus. Une permanence de la violence, qui finit par se jouer tant des registres du public et du privé que de ceux du mobile et de l'immobile, traduisant l'indistinction des configurations dans laquelle elle sévit.

L'auteur souligne les conditions et les situations de départ de ces femmes, point encore peu soulevé dans un champ d'études sur les migrations féminines souvent focalisé sur les populations immigrées. Car si le voyage clandestin constitue bien souvent une succession de violences de toute sorte, pouvant parfois conduire à un "phénomène d'indistinction ", les différentes situations de vulnérabilité et de dépendance des femmes sur la route peuvent également être analysées sous l'angle des différentes positions sociales dans les pays d'origine. Et l'auteur de rappeler que les femmes connaissant une faible insertion sociale et économique dans leur pays d'origine seront davantage exposées à la violence du parcours, celle-ci les enfermant alors dans une "répétition sans fin du malheur ».

Le Sahara franchi, l'auteur entrevoit le vécu de ces femmes en prise avec un contexte sociétal maghrébin où les hypothétiques protections et recours s'articulent sur des politiques étatiques arbitraires. Le sociologue précise ainsi les contextes sociétaux des pays du Maghreb en matière d'immigration et de droit d'asile, en rappelant que les questions de production des catégories institutionnelles se situent à l'interface de politiques de sécurité et de relations intéressées et complexes avec l'Union Européenne. Dans un contexte de recherche de sécurité, le refus politique de l'asile s'exprime alors dans le rapprochement entre la condition d'immigré clandestin et celle de demandeur d'asile.

En appréhendant les rapports avec l'institution à travers l'analyse des récits des migrantes, l'auteur met à jour les décalages entre les codes institutionnels et les expériences féminines réelles de la violence. En identifiant l' " histoire officielle »-via les invariants autobiographiques - présentée par des femmes du Centre de séjour temporaire de Melilla, le sociologue dévoile quelquesuns des effets néfastes que prennent les stratégies d'audibilité face à une institution à la recherche de violences spécifiques et juridiquement définies.

La construction de ces «histoires officielles» participent au processus d'invisibilisation des violences vécues, mais surtout entravent l'appréhension des migrantes comme actrices de leurs projets migratoires. La demande d'asile et son corollaire de preuve des persécutions subies amènent leur lot de nouvelles difficultés inhérentes à la fois à l'exposition des violences subies, et à l'identification et à l'évaluation de la valeur de la persécution. Dans cette perspective, les acteurs institutionnels continuent à trancher sur ce qui leur parait vraisemblable plutôt que de décloisonner et d'autonomiser les différents univers (économiques, politiques, religieux, etc.) souvent présentés en bloc par les migrantes au cours de leurs récits. Taux de refus et solidarité des refoulés se développent alors de pair, traduisant une 
mise à distance interdisant l'accès à une certaine « responsabilité du monde ».

À travers cet ouvrage, le sociologue Smaïn Laacher se saisit de la question encore non traitée de la violence faite aux femmes migrantes lors de leur «voyage clandestin ». Il entreprend notamment cette nouvelle voie de recherche par la restitution de larges passages d'entretiens que Smaïn Laacher entreprend un zoom important et nécessaire sur des conditions féminines africaines contemporaines sur les routes migratoires transsahariennes. Les situations d'animosité qu'elles peuvent décider de fuir ne signifient que rarement le basculement dans un monde meilleur. Á la position sociale dans les territoires de départ fait suite la condition de voyageuse, et à la condition de voyageuse fait suite celle de "figure trouble ». Ainsi, tandis que les registres de violences physiques et morales se succèdent, la violence des catégories vient s'y s'ajouter comme levier d'action d'une politique d'asile européenne toujours plus bâtisseuse de murs.
Mickaël Quintard Doctorant en Géographie Migrinter - UMR 7301 CNRS / Université de Poitiers mickael.quintard@univ-poitiers.fr 